\title{
Investigation of velocity field and oil distribution in an oil-water hydrocyclone using a particle dynamics analyzer
}

\author{
Ningyu Zhou ${ }^{\mathrm{a}, \mathrm{b}}$, Yingxin Gao ${ }^{\mathrm{a}}$, Wei An ${ }^{\mathrm{a}}$, Min Yang ${ }^{\mathrm{a}, *}$ \\ a State Key Laboratory of Environmental Aquatic Chemistry, Research Center for Eco-Environmental Sciences, \\ Chinese Academy of Sciences, P.O. Box 2871, 18 Shuangqing Road, Haidian District, Beijing 100085, China \\ b Logistic Engineering University, Chongqing 400016, China
}

\section{A R T I C L E I N F O}

\section{Article history:}

Received 13 May 2009

Received in revised form 19 October 2009

Accepted 27 October 2009

\section{Keywords:}

Hydrocyclone

Particle dynamics analyzer (PDA)

Velocity

Concentration

Oil droplet size

\begin{abstract}
A B S T R A C T
A non-intrusive particle dynamics analyzer (PDA) was used to investigate the distribution of velocity field and oil droplets in a $50 \mathrm{~mm}$ oil-water separation hydrocyclone. Axi-asymmetry distribution of the flow field was observed in the hydrocyclone, which was perhaps attributed to the use of single tangential inlet in the hydrocyclone. The average size of oil droplets decreased from $43-126 \mu \mathrm{m}$ at $Z=0 \mathrm{~mm}$ (the top) to $19-21 \mu \mathrm{m}$ at $Z=990 \mathrm{~mm}$ (the bottom) at an inlet flow rate of $2000 \mathrm{~L} / \mathrm{h}$ and flow rejection ratio of $20 \%$. The average size of oil droplets at $Z=990 \mathrm{~mm}$ decreased from approximately $40-20 \mu \mathrm{m}$ with the increase of flow rate from 1300 to $2000 \mathrm{~L} / \mathrm{h}$, showing the important impacts of the inlet flow rate on the removal of small oil droplets by the hydrocyclone. The concentration distribution of oil droplets in the upper cylindrical sections was characterized by the existence of a "concentration valley" surrounding the oil core. The "concentration valley" disappeared from $Z=590 \mathrm{~mm}$. Results show that PDA is a powerful instrument for the characterization of oil-water separation hydrocyclones because it can present the velocity field and the distribution of oil droplets in the hydrocyclone simultaneously.
\end{abstract}

(c) 2009 Elsevier B.V. All rights reserved.

\section{Introduction}

Efficient oil-water separation is often required in the petroleum and other industries. Hydrocyclone technology provides a competitive method for the treatment of oily water [1], due to its absence of moving parts and chemical additives, and its compactness [2-4]. Efforts have been made to study the relationship between the oil removal efficiency and the dimensional and operational variables of hydrocyclones $[2,4,5]$. For optimizing the structure and improving the efficiency of oil-water separation hydrocyclones, however, it is also important to understand the changes of fluid flow field and the size distribution of oil droplets within the devices under different conditions.

The first description of the velocity profile of a hydrocyclone was done by Kelsall in 1952 [6]. With the fast development of Laser Doppler Velocimetry (LDV), it became much easier to determine the flow field in solid-liquid or oil-water separation hydrocyclones [7-12]. Among the three velocity components, tangential velocity has been found to govern the flow field and to be closely related to the performance of a hydrocyclone [13-16]. LDV, however, is unable to provide information on particle distribution within the

\footnotetext{
* Corresponding author. Tel.: +86 10 62923475; fax: +86 1062923541 .

E-mail address: yangmin@rcees.ac.cn (M. Yang).
}

hydrocyclone. Particle dynamics analyzer (PDA), which is an extension of the LDV technique, has recently been used to measure the motion of particles and ozone bubbles in water treatment reactors [17-19]. This analyzer, however, has not yet been applied to the analysis of size distribution of oil droplets in an oil-water hydrocyclone.

The objective of this study was to determine the flow field and the distribution of oil droplets in an oil-water hydrocyclone simultaneously using a PDA. The results are useful to better understand oil-water separation behavior in a hydrocyclone, and could be used for the validation of simulation results, which are essential for the optimization of hydrocyclone configurations.

\section{Experimental methods}

\subsection{Hydrocyclone}

A glass hydrocyclone with design parameters recommended by Young et al. [5] was constructed as shown in Fig. 1a. The hydrocyclone consisted of two cylindrical sections and one conical section. The inlet $(13 \mathrm{~mm}$ in diameter, D1) and overflow orifice $(8 \mathrm{~mm}$ in diameter, D3) were attached to the upper cylindrical section, which had a diameter (D) of $50 \mathrm{~mm}$ and a length (L1) of $50 \mathrm{~mm}$. The outlet $(19 \mathrm{~mm}$ in diameter) was attached to the lower cylindrical section (D2, $19 \mathrm{~mm}$; L3, $900 \mathrm{~mm}$ ). The conical section connecting 
(a)

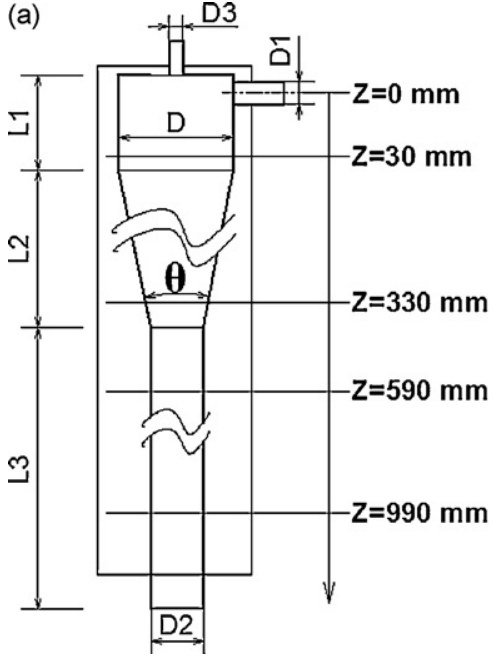

(b)

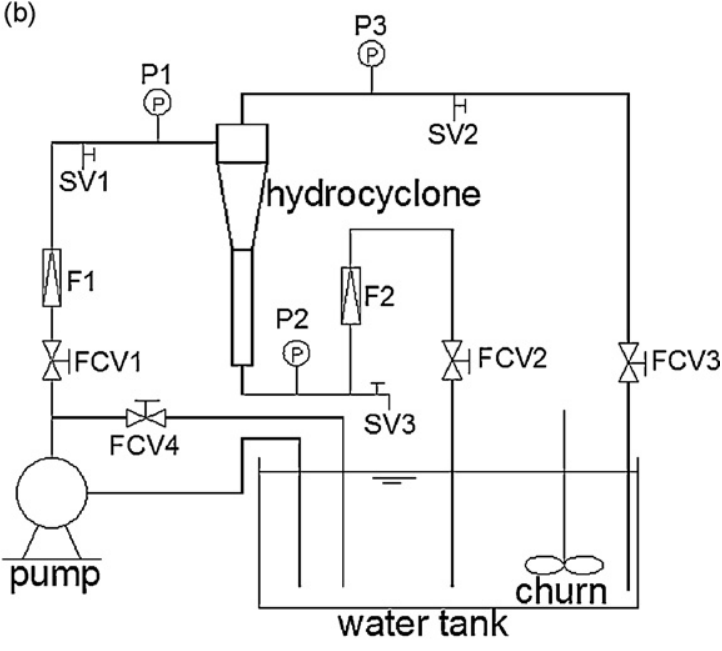

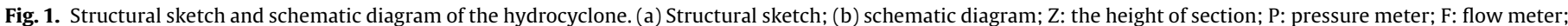
FCV: flow control valve.

the upper and lower cylindrical sections had a cone angle $\theta$ of $5.5^{\circ}$, and a length (L2) of $322 \mathrm{~mm}$. In order to minimize the optical refraction of the laser beams at the curved hydrocyclone wall, the hydrocyclone was covered with a rectangular optical compensating box $(100 \mathrm{~mm} \times 100 \mathrm{~mm})$. The space between the box and the hydrocyclone was filled with mineralized water, which was prepared by adding inorganic salts to deionized water as follows: $\mathrm{NaCl}$ $1.513 \mathrm{~g} / \mathrm{L}, \mathrm{KCl} 0.075 \mathrm{~g} / \mathrm{L}, \mathrm{MgCl}_{2} \cdot 6 \mathrm{H}_{2} \mathrm{O} 0.097 \mathrm{~g} / \mathrm{L}, \mathrm{Na}_{2} \mathrm{SO}_{4} 0.04 \mathrm{~g} / \mathrm{L}$, $\mathrm{Na}_{2} \mathrm{CO}_{3} 1.867 \mathrm{~g} / \mathrm{L}$ and $\mathrm{NaHCO}_{3} 3.356 \mathrm{~g} / \mathrm{L}$.

\subsection{Experimental setup}

The schematic diagram of the experimental apparatus is illustrated in Fig. 1b. Oil-containing water, which was used as the working fluid, was prepared by dissolving lubricating oil $(0.86 \mathrm{~g} / \mathrm{mL})$ in the mineralized water to give an oil concentration of approximately $40 \mathrm{mg} / \mathrm{L}$. The diameter of the oil droplets was determined to be $0.4-158 \mu \mathrm{m}$. These oil droplets provided sufficient flow seeding, which is required for laser velocimetry. The oil-containing water was pumped from a 200 -L water tank to the inlet of the hydrocyclone, and was then split into two streams: the oil-rich overflow stream and the oil-poor underflow stream. The two streams were finally returned to the same water tank. A flow control valve (FCV4) was installed in a bypass pipe to regulate the inlet flow rate $(Q)$. Because the hydrocyclone was made of glass, the highest inlet flow rate was restricted to be $2000 \mathrm{~L} / \mathrm{h}$. The flow rejection ratio $(R)$, which is defined as the ratio of the overflow rate to the inlet flow rate $\left(\left(Q_{\text {overflow }} / Q\right) \times 100 \%\right)$ [1] was set over $20 \%$ to control the refraction caused by the air core [10]. The temperature of the oil-containing water was $22-24^{\circ} \mathrm{C}$.

\subsection{Measurements}

A non-intrusive PDA (58N50, Dantec Co., Denmark) was used to measure the flow field and the size and concentration distributions of oil droplets in the tested hydrocyclone (Fig. 2), and some technical parameters of the PDA system are given in Table 1 . The velocity was acquired from the frequency of the Doppler burst, and the tangential velocity was given by resolving the measured velocity using a Cartesian coordinate system. The size measurement is based on comparing the signals from multiple detectors located at different scattering angles. Oil droplet concentration was expressed as the number of oil droplets/mL. At each measurement point, 1500 samples were collected to ensure the validity of the measurements,

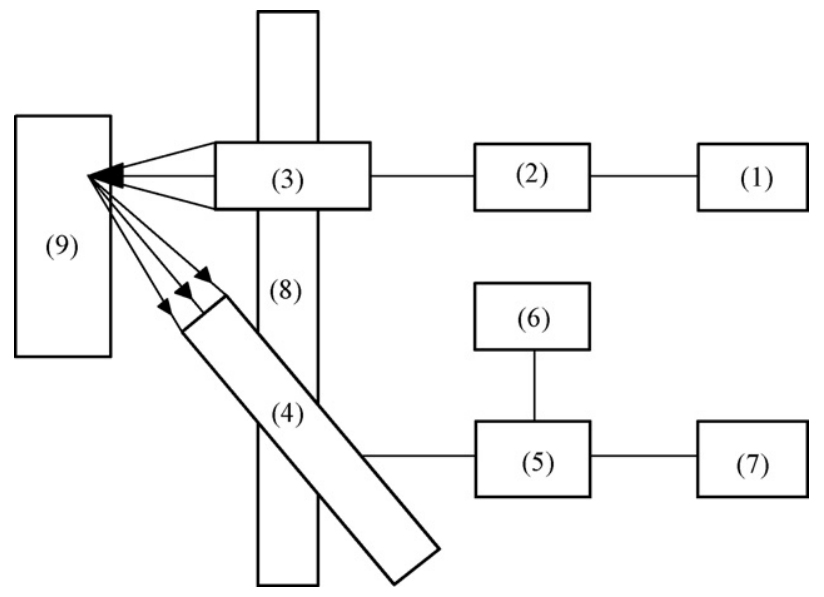

Fig. 2. PDA system schematic diagram. (1) Laser producer; (2) transmitting optics (3) laser probe; (4) signal receiving optics; (5) signal processor; (6) oscilloscope; (7) computer; (8) movable mounting bench; (9) hydrocyclone.

with a time limit of 2 min. The sections at which measurements were conducted are shown in Fig. 1a.

\section{Results and discussion}

\subsection{Tangential velocity in the hydrocyclone}

Fig. 3a shows the tangential velocities at different sections at an inlet flow rate of $1300 \mathrm{~L} / \mathrm{h}$ and flow rejection ratio of $20 \%$. The radial position was made dimensionless by dividing the distance of the position from the center by the radius of the section. It can be seen from the figure that the tangential velocity increased rapidly to a maximum with increasing radial distance from the axis, and then decreased slowly, presenting the characteristics of a forced vortex near the axis, and a free vortex in the outer portion, with a transition zone in between. The observations are similar to

Table 1

Technical parameters of the PDA system.

\begin{tabular}{llll}
\hline Measurement & Velocity $(\mathrm{m} / \mathrm{s})$ & Size $(\mu \mathrm{m})$ & $\begin{array}{l}\text { Concentration (number } \\
\text { of oil droplets/mL) }\end{array}$ \\
\hline Range & $0-500$ & $0.5-10,000$ & $0-10^{6}$ \\
Accuracy & $1 \%$ & $4 \%$ & $30 \%$ \\
\hline
\end{tabular}



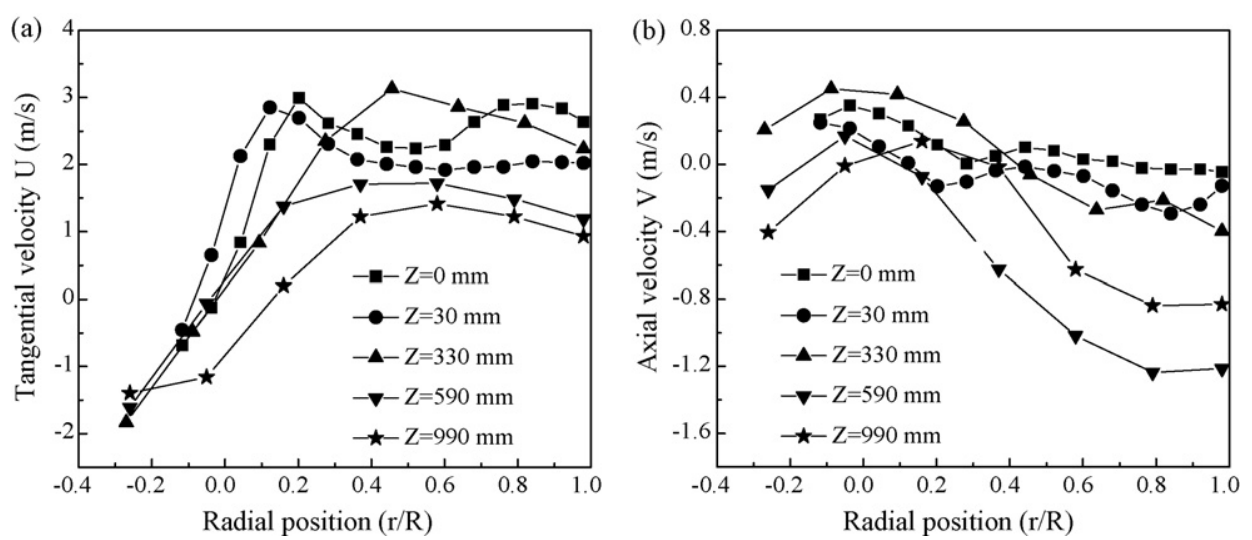

Fig. 3. Variations in tangential and axial velocities along the hydrocyclone height. Inlet flow rate: $1300 \mathrm{~L} / \mathrm{h}$; rejection ratio: $20 \%$; (a) tangential velocity; (b) axial velocity.

previous experimental studies with LDV $[8,9,20]$. With an LDV, Zhishan et al. found that the maximum tangential velocity decreased from the top to the bottom in an oil-water separation hydrocyclone [12]. Bhaskar et al. acquired a similar result through CFD modeling [21]. In this study, however, the highest maximum tangential velocity occurred at $Z=330 \mathrm{~mm}$ in the conical section, which was in accordance with that observed by Chine and Concha with an LDV [8]. It is speculated that the above discrepancies were attributed to the differences of the conical configuration in different hydrocyclones. So optimization of the conical configuration is important to maintain a relatively high tangential velocity in the hydrocyclone.

Fig. 3a also shows that the position of zero tangential velocity was not always located at the geometrical center of hydrocyclone,
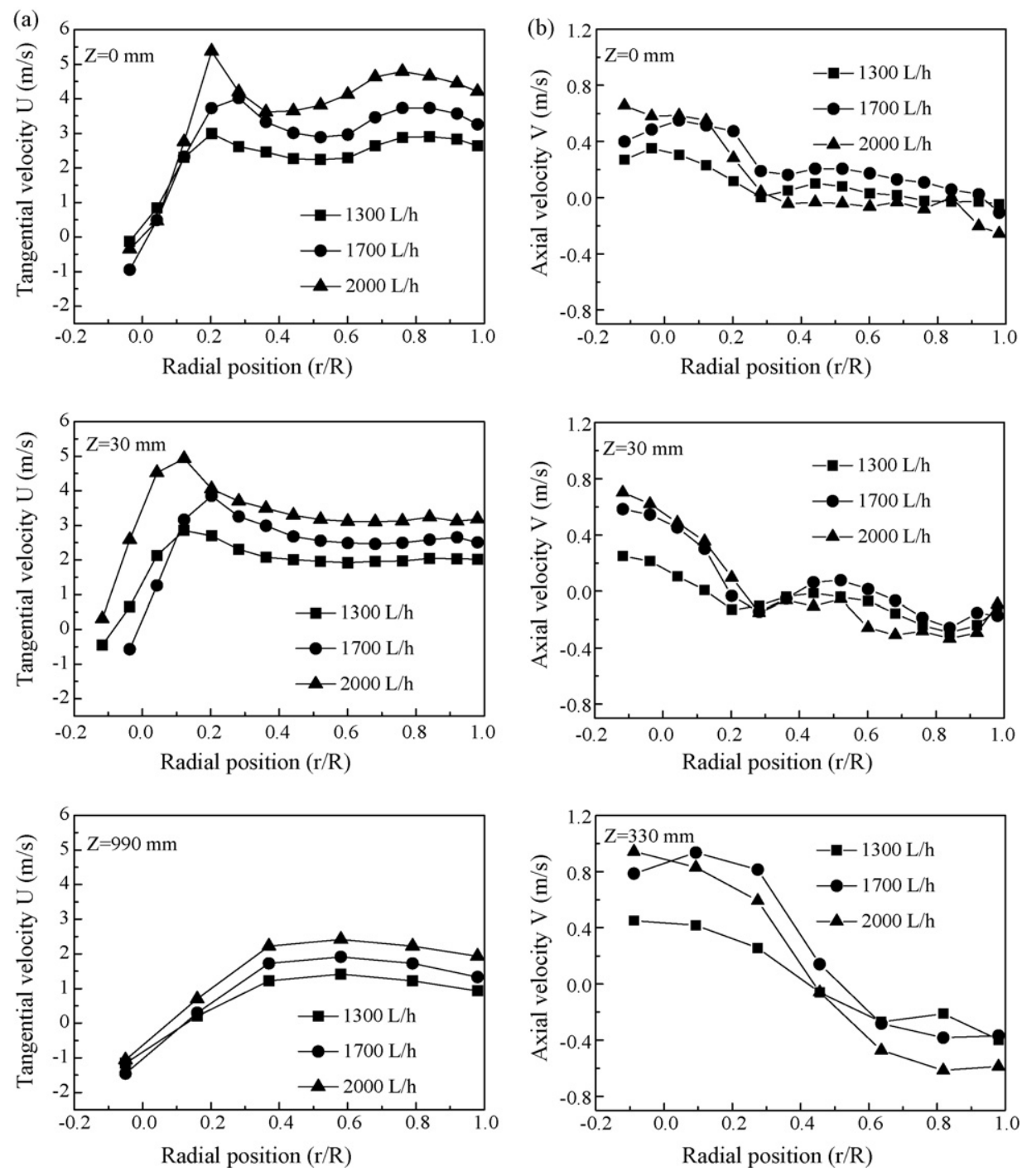

Fig. 4. Effects of inlet flow rate on tangential and axial velocities. Rejection ratio: $20 \%$; (a) tangential velocity; (b) axial velocity. 

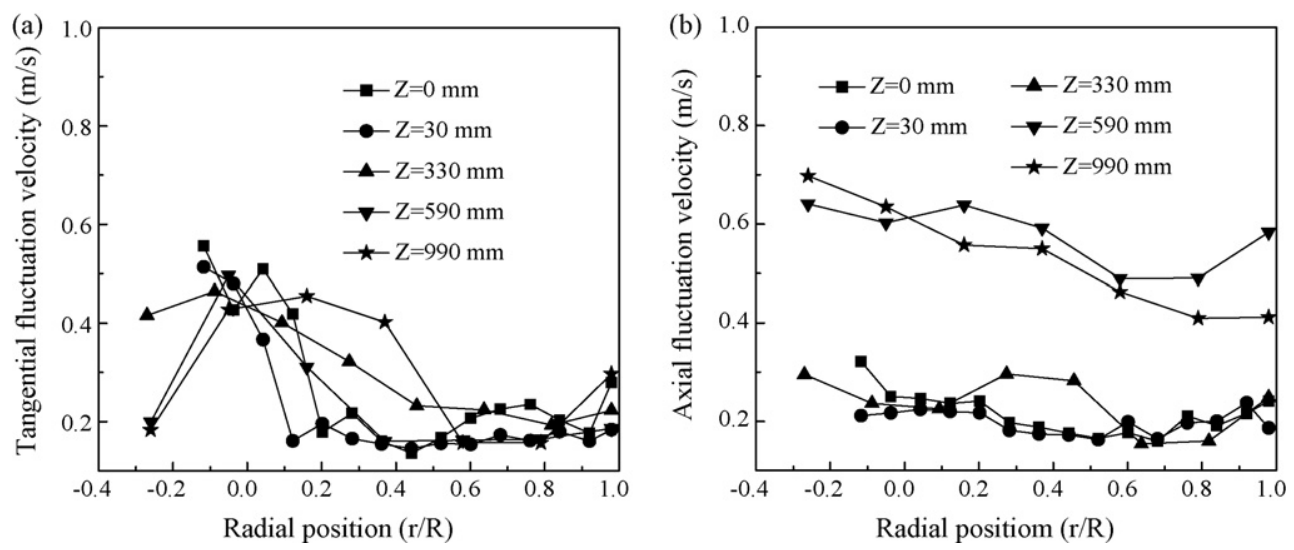

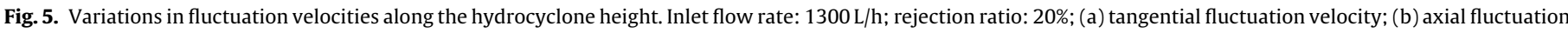
velocity.

indicating that the flow field in the hydrocyclone was not an axis symmetry distribution, which was also observed in a previous study [20]. The axi-asymmetry distribution of the flow field in the hydrocyclone was perhaps attributed to the use of single tangential inlet in the hydrocyclone.

The effects of inlet flow rate on tangential velocity are shown in Fig. 4a. The tangential velocity increased with the increase of inlet flow rate, but the profile of tangential velocity in each section did not changes, which was similar to previous experimental studies with LDV $[8,12]$.

\subsection{Axial velocity in the hydrocyclone}

Fig. 3b shows the axial velocities at different sections at an inlet flow rate of $1300 \mathrm{~L} / \mathrm{h}$ and flow rejection ratio of $20 \%$. It can be observed from the figure that there were downward flow (axial velocity was negative) close to the hydrocyclone wall and upward flow (axial velocity was positive) near the center. The highest maximum axial velocity of upward flow occurred at $Z=330 \mathrm{~mm}$ in the conical section. In the cylindrical section, the positive values of axial velocity decreased with increasing axial distance. At the axial height of $990 \mathrm{~mm}$, the axial velocity near the center was positive, indicating that further oil-water separation happened in this region.

The effects of inlet flow rate on axial velocity are shown in Fig. 4b. It can be seen from Fig. 4b that the inlet flow rate influenced mainly the upward flow. The maximum value of positive axial velocity increased with the increase of inlet flow rate, and the flow characters were not changed by changes in the inlet flow rate.

\subsection{Fluctuation velocity in the hydrocyclone}

Fig. 5 shows the fluctuation velocities at different sections at an inlet flow rate of $1300 \mathrm{~L} / \mathrm{h}$ and flow rejection ratio of $20 \%$. The maximum tangential fluctuation velocity at each section was observed near the center (Fig. 5a), which might be caused by the greater tangential velocity grads near the center. Fig. 5b shows that the axial fluctuation velocity decreased from the hydrocyclone wall towards the center, but began to increase after reaching a minimum value. The axial fluctuation velocity in the lower cylindrical sections was greater than that in the upper cylindrical and conical sections. The effects of inlet flow rate on fluctuation velocities are shown in Fig. 6. The fluctuation velocities increased with the increase of inlet flow rate.

\subsection{Size distribution of oil droplets in the hydrocyclone}

Fig. 7 illustrates the average size distribution of oil droplets along the radial direction at different sections of the hydrocyclone, under a given operating condition $(Q=2000 \mathrm{~L} / \mathrm{h}, R=20 \%)$. With the decrease of radial distance from 1 to 0.1 , the average size of the oil droplets increased from 43 to $126 \mu \mathrm{m}$, from 25 to $88 \mu \mathrm{m}$, from 25 to $38 \mu \mathrm{m}$, and from 19 to $21 \mu \mathrm{m}$, at $Z=0 \mathrm{~mm}, Z=30 \mathrm{~mm}, Z=590 \mathrm{~mm}$, and $Z=990 \mathrm{~mm}$, respectively. This result demonstrates that large oil droplets tend to move to the center of the hydrocyclone, which is opposite to that for solid-liquid separation [22]. The large oil droplets that accumulated around the center of the hydrocyclone could be taken out from the top through the overflow orifice, so the average size of the oil droplets decreased from the top to the
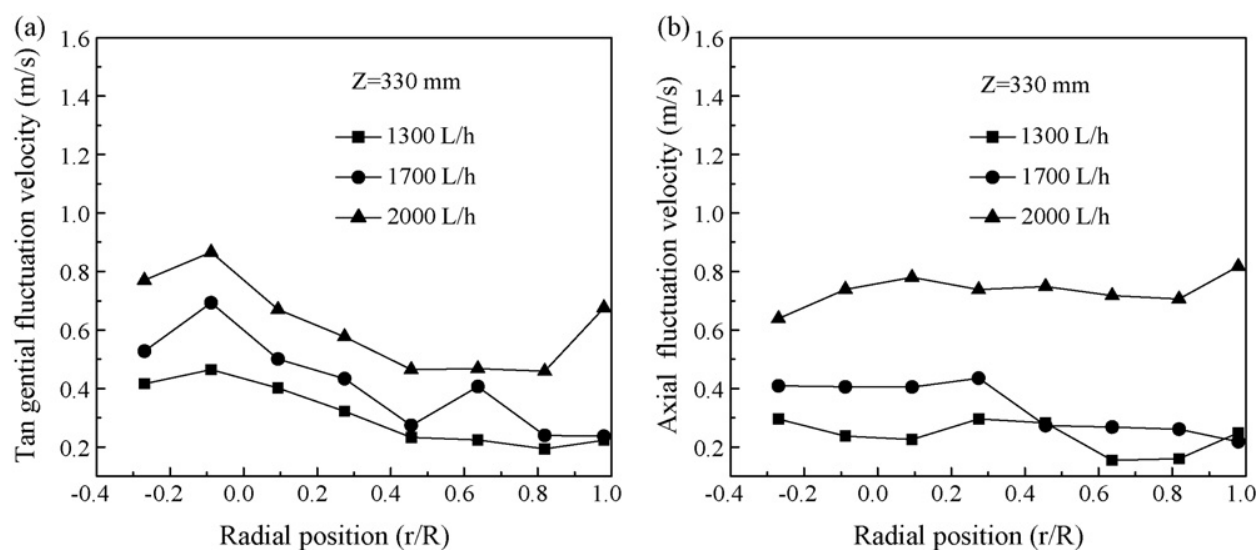

Fig. 6. Effects of inlet flow rate on fluctuation velocity. Rejection ratio: 20\%; (a) tangential fluctuation velocity; (b) axial fluctuation velocity. 


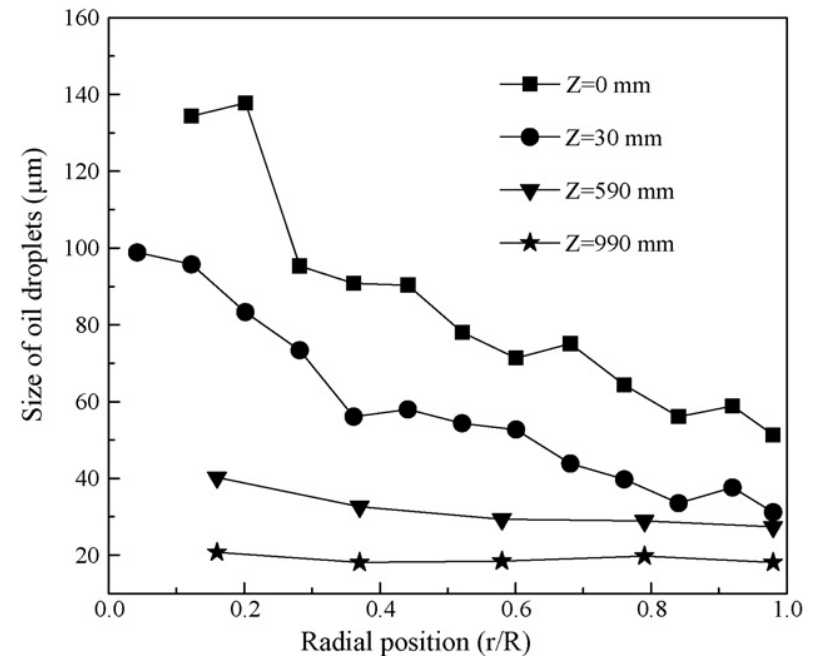

Fig. 7. Distribution of oil droplets along the hydrocyclone height. Inlet flow rate: $2000 \mathrm{~L} / \mathrm{h}$; rejection ratio: $20 \%$.

bottom, for a given radial position. At the section of $Z=990 \mathrm{~mm}$, the average oil droplet size only changed from 19 to $21 \mu \mathrm{m}$ from the wall toward the axis, indicating that the hydrocyclone was not effective in removing oil droplets with an average size smaller than $20 \mu \mathrm{m}$.

Fig. 8 shows the influence of inlet flow rate $(1300,1700$ and $2000 \mathrm{~L} / \mathrm{h}$ ) on the average size distribution of oil droplets at $Z=990 \mathrm{~mm}(R=20 \%)$. It is clear that the radial changes of the average size of oil droplets at $Z=990 \mathrm{~mm}$ were negligible, and the average size decreased from approximately $40-20 \mu \mathrm{m}$ with an increase in inlet flow rate from 1300 to $2000 \mathrm{~L} / \mathrm{h}$, corresponding to the increase of the maximum tangential velocity from 1.41 to $2.42 \mathrm{~m} / \mathrm{s}$. This result shows that the inlet flow rate has a significant impact on the size distribution of oil droplets, and it is possible to further reduce the oil droplet size in the effluent by increasing the inlet flow rate.

Fig. 9 shows the influence of flow rejection ratio (20\%,30\% and $40 \%$ ) on the average size distribution of oil droplets at $Z=0 \mathrm{~mm}$, $Z=30 \mathrm{~mm}$ and $Z=590 \mathrm{~mm}$. Fig. 9 a shows that at $Z=0 \mathrm{~mm}$, with the decrease of radial distance from 1 to 0.1 , the average size of the oil droplets increased from 53.3 to $137.8 \mu \mathrm{m}$, from 53.2 to $96.8 \mu \mathrm{m}$, and from 50.5 to $86.0 \mu \mathrm{m}$ for the flow rejection ratios of $20 \%, 30 \%$ and $40 \%$, respectively. The average size distributions of oil droplets

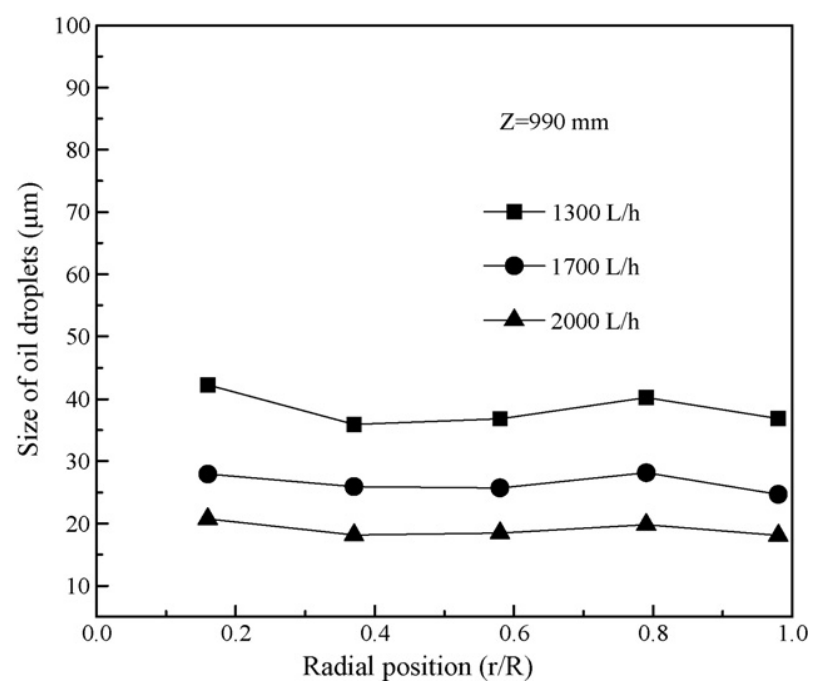

Fig. 8. Influence of inlet flow rate on the distribution of oil droplet size. Rejection ratio: $20 \%$. at $Z=30 \mathrm{~mm}$ and $Z=590 \mathrm{~mm}$ (Fig. $9 \mathrm{~b}$ and $\mathrm{c}$ ) also show similar trends. This is why the oil removal was found to increase with the increase of flow rejection ratio [1].

\subsection{Concentration distribution of oil droplets in the hydrocyclone}

The concentration distributions of oil droplets expressed as the number of oil droplets/mL under different inlet flow rates are presented in Fig. 10. It can be seen that in the upper cylindrical sections, the concentration of small oil droplets at the radial position near the wall is higher than that near the center, while the concentration of large oil droplets at the radial position near the wall is lower than that near the center. The oil droplets near the center
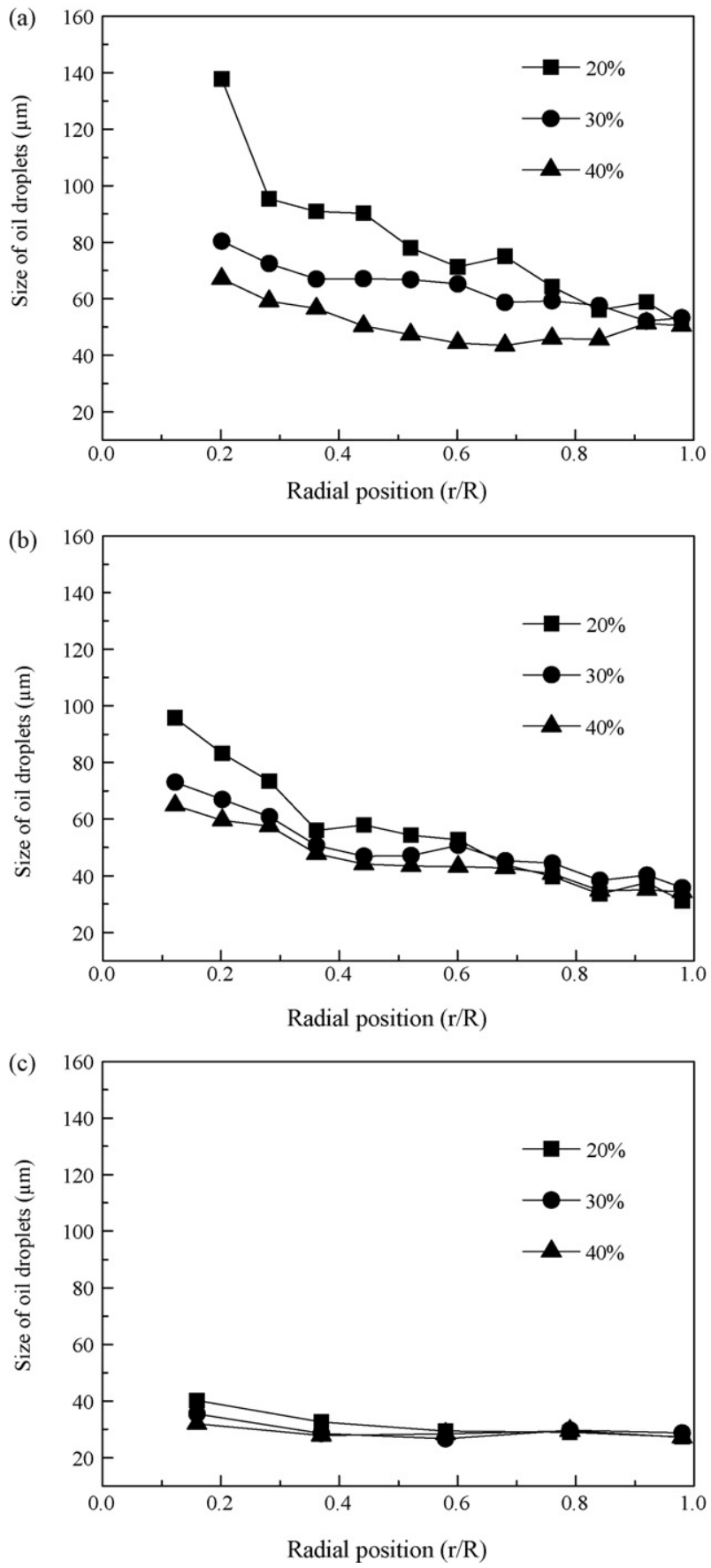

Fig. 9. Influence of flow rejection ratio on the distribution of oil droplet size. Inlet flow rate: $2000 \mathrm{~L} / \mathrm{h}$; (a) $Z=0 \mathrm{~mm}$; (b) $Z=30 \mathrm{~mm}$; (c) $Z=590 \mathrm{~mm}$. 
(a)
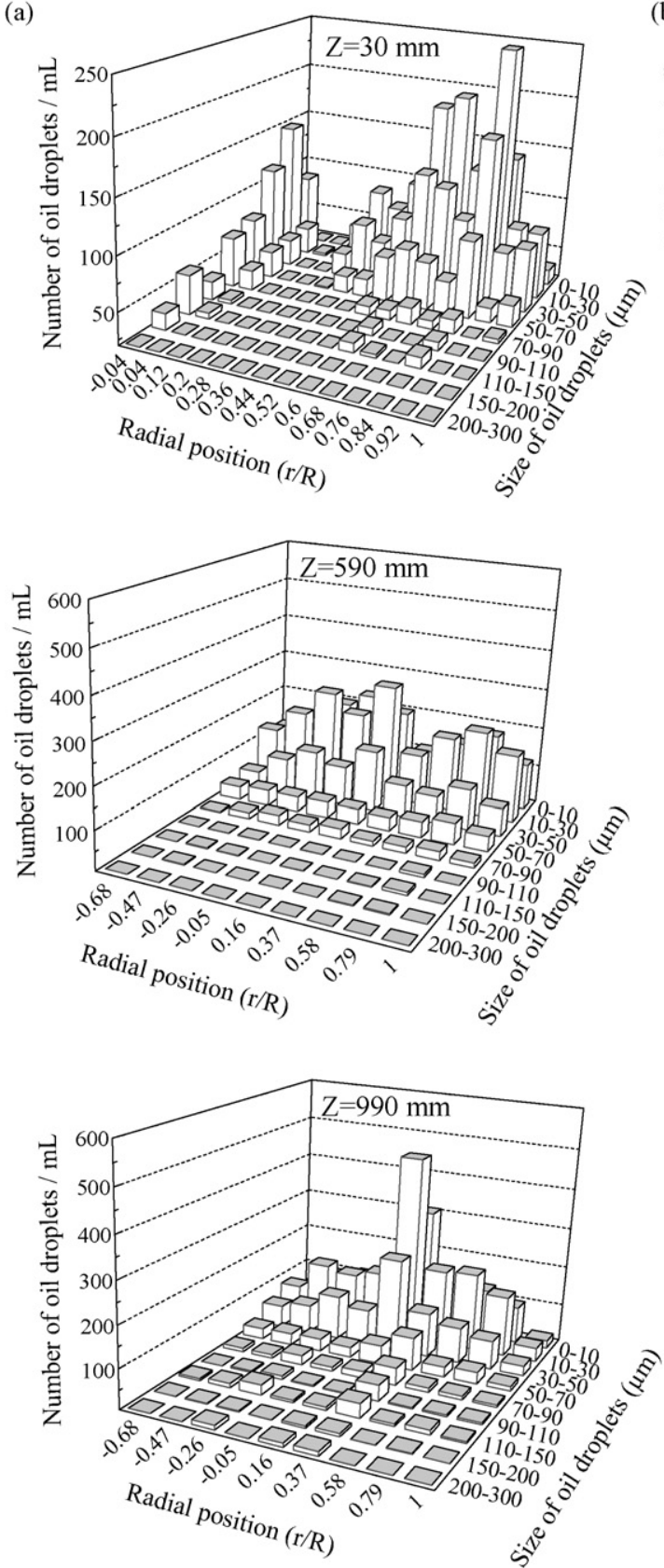

(b)
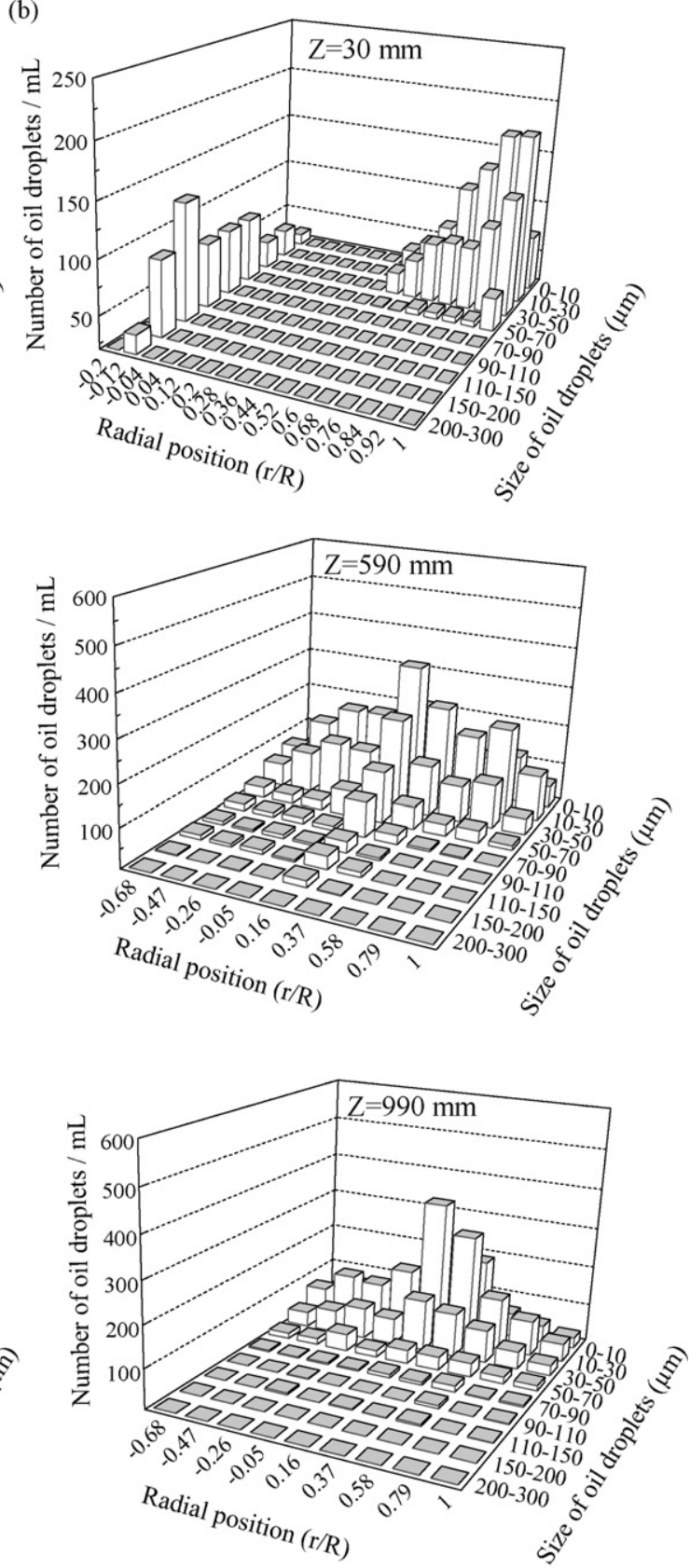

Fig. 10. Concentration distribution of oil droplets in the hydrocyclone. (a) Inlet flow rate: $1300 \mathrm{~L} / \mathrm{h}$; rejection ratio: $20 \%$; (b) inlet flow rate: $2000 \mathrm{~L} / \mathrm{h}$; rejection ratio: $20 \%$.

form a narrow oil core along the axis. The oil droplet concentration surrounding the oil core was relatively low, forming a "concentration valley" in the upper sections. This phenomenon has not been previously reported. In comparison with the low flow rate condition $(1300 \mathrm{~L} / \mathrm{h})$, the high flow rate condition $(200 \mathrm{~L} / \mathrm{h})$ presented a much bigger "concentration valley". The effects of such a "concentration valley" on oil-water separation, however, need to be further studied.

The "concentration valley" was absent from the section of $Z=590 \mathrm{~mm}$. In the lower cylindrical sections, the concentration of oil droplets increased with decreasing radial distance, reaching a maximum value near the center of the hydrocyclone. It is clear that concentration of small oil droplets $(0-10 \mu \mathrm{m})$ near the axis of the hydrocyclone still occurred at the section of $Z=990 \mathrm{~mm}$, suggesting the existence of relatively strong centrifugal force at the bottom. However, the concentrated oil droplets near the axis could not be taken out because the axial velocity at the section was below zero. So it is important to take measures to increase the axial velocity for improving the oil-water separation efficiency.

As shown in Fig. 10, the concentration of small oil droplets in the oil core decreased along the hydrocyclone height from the bottom to the top, while the concentration of large oil droplets increased. This phenomenon might be attributed to the aggregation of oil droplets in the oil core, due to their high concentration.

\section{Conclusion}

By using a non-intrusive particle dynamics analyzer (PDA), it became possible to measure the velocity field and the distribution of oil droplets in an oil-water separation hydrocyclone simultaneously, which was very important to better understand the oil-water separation process. The following conclusions were acquired. 
(1) Particle dynamics analyzer (PDA) can effectively measure the velocity field and the concentration field in an oil-water hydrocyclone simultaneously.

(2) The fluctuation velocities increased with the increase of inlet flow rate, and the maximum fluctuation velocities appeared at the center of the upward flow.

(3) The average oil droplet diameter shows decreasing trend from the axis toward the wall, and it decreased from $43-126 \mu \mathrm{m}$ at $Z=0 \mathrm{~mm}$ (the top) to $19-21 \mu \mathrm{m}$ at $Z=990 \mathrm{~mm}$ (the bottom) at an inlet flow rate of $2000 \mathrm{~L} / \mathrm{h}$ and flow rejection ratio of $20 \%$. The average size of the oil droplets at $Z=990 \mathrm{~mm}$ decreased from approximately 40 to $20 \mu \mathrm{m}$ with an increase in inlet flow rate from 1300 to $2000 \mathrm{~L} / \mathrm{h}$.

(4) The average size distribution of oil droplets decreases with the increases of the flow rejection ratio.

(5) The concentration distribution of oil droplets in the upper cylindrical sections was characterized by the existence of a "concentration valley" surrounding the oil core. The "concentration valley" was absent from the section at $Z=590 \mathrm{~mm}$.

\section{Acknowledgements}

This work was supported by the National Natural Science Foundation of China (Nos. 50621804, 50525824). Support for this work by Key Laboratory for Thermal Science and Power Engineering of Ministry of Education, Tsinghua University is gratefully appreciated.

\section{References}

[1] T. Husveg, O. Rambeau, T. Drengstig, T. Bilstad, Performance of a deoiling hydrocyclone during variable flow rates, Miner. Eng. 20 (2007) 368-379.

[2] A. Belaidi, M.T. Thew, S.J. Mumaweera, Hydrocyclone performance with complex oil-water emulsions in the feed, Can. J. Chem. Eng. 81 (2003) 1159-1170.

[3] S. Huang, Numerical simulation of oil-water hydrocyclone using Reynoldsstress model for eulerian multiphase flows, Can. J. Chem. Eng. 83 (2005) 829-834.

[4] C. Gomez, J. Caldenfey, S. Wang, L. Gomez, R. Mohan, O. Shoham, Oil/water separation in liquid/liquid hydrocyclones (LLHC). Part 1. Experimental investigation, SPE J. 7 (2002) 353-361.
[5] G.A.B. Young, W.D. Wakley, D.L. Taggart, S.L. Andrews, J.R. Worrell, Oil-water separation using hydrocyclones: an experimental search for optimum dimensions, J. Petrol. Sci. Eng. 11 (1994) 37-50.

[6] D.F. Kelsall, A study of the motion of solid particles in a hydraulic cyclone, Trans. Inst. Chem. Eng. 30 (1952) 87-108.

[7] J. Collantes, F. Concha, B. Chine, Axial symmetric flow model for a flat bottom hydrocyclone, Chem. Eng. J. 80 (2000) 257-265.

[8] B. Chine, F. Concha, Flow patterns in conical and cylindrical hydrocyclones, Chem. Eng. J. 80 (2000) 267-273.

[9] W. Peng, P.J.A.J. Boot, A.C. Hoffmann, H.W.A. Dries, J. Kater, A. Ekker, Flow in the inlet region in tangential inlet cyclones, Ind. Eng. Chem. Res. 40 (2001) 5649-5655.

[10] M.J. Fisher, R.D. Flack, Velocity distributions in a hydrocyclone separator, Exp. Fluids 32 (2002) 302-312.

[11] M.J. Doby, A.F. Nowakowski, E. Nowak, T. Dyakowski, Numerical and experimental examination of swirl flow in a cylindrical container with rotating lids, Miner. Eng. 20 (2007) 361-367.

[12] B. Zhi-shan, W. Hua-lin, T. Shan-tung, Experimental study of flow patterns in deoiling hydrocyclone, Miner. Eng. 22 (2009) 319-323.

[13] J. Bergstrom, H. Vomhoff, Velocity measurements in a cylindrical hydrocyclone operated with an opaque fiber suspension, Miner. Eng. 17 (2004) 599-604.

[14] S. Murphy, R. Delfos, M.J.B.M. Pourquie, Z. Olujic, P.J. Jansens, F.T.M. Nieuwstadt, Prediction of strongly swirling flow within an axial hydrocyclone using two commercial CFD codes, Chem. Eng. Sci. 62 (2007) 1619-1635.

[15] C. Puprasert, G. Hebrard, L. Lopez, Y. Aurelle, Potential of using hydrocyclone and hydrocyclone equipped with grit pot as a pre-treatment in run-off water treatment, Chem. Eng. Process. 43 (2004) 67-83.

[16] K.U. Bhaskar, Y.R. Murthy, M.R. Raju, S. Tiwari, J.K. Srivastava, N. Ramakrishnan, CFD simulation and experimental validation studies on hydrocyclone, Miner. Eng. 20 (2007) 60-71.

[17] H. Zhou, D.W. Smith, Ozone mass transfer in water and wastewater treatment: experimental observations using a 2D laser particle dynamics analyzer, Water Res. 34 (2000) 909-921.

[18] L.Y. Chu, W.M. Chen, X.Z. Lee, Effects of geometric and operating parameters and feed characters on the motion of solid particles in hydrocyclones, Sep. Purif. Technol. 26 (2002) 237-246.

[19] Y. Su, The turbulent characteristics of the gas-solid suspension in a square cyclone separator, Chem. Eng. Sci. 61 (2006) 1395-1400.

[20] J. Bergstrom, H. Vomhoff, D. Soderberg, Tangential velocity measurements in a conical hydrocyclone operated with a fibre suspension, Miner. Eng. 20 (2007) 407-413.

[21] K.U. Bhaskar, Y.R. Murthy, N. Ramakrishnan, J.K. Srivastava, S. Sarkar, V. Kumar, CFD validation for flyash particle classification in hydrocyclones, Miner. Eng. 20 (2007) 290-302.

[22] L.Y.Chu, W.M. Chen, Research on the motion of solid particles in a hydrocyclone, Sep. Sci. Technol. 28 (1993) 1875-1886. 